\title{
Surface Piezoelectricity and Pyroelectricity in Centrosymmetric Materials: A Case of $\alpha$-Glycine
}

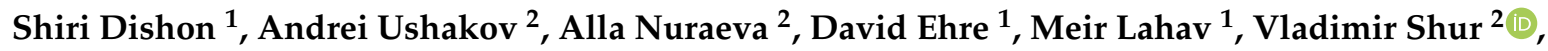 \\ Andrei Kholkin $2,3, * \mathbb{1}$ and Igor Lubomirsky ${ }^{1, *}$ \\ 1 Department of Materials and Interfaces, Weizmann Institute of Science, Herzl St 234, Rehovot 7610001, Israel; \\ shiri.dishon@weizmann.ac.il (S.D.); david.ehre@weizmann.ac.il (D.E.); meir.lahav@weizmann.ac.il (M.L.) \\ 2 School of Natural Sciences and Mathematics, Ural Federal University, Lenin Ave. 51, \\ 620000 Ekaterinburg, Russia; andrey.ushakov@urfu.ru (A.U.); alla.nuraeva@urfu.ru (A.N.); \\ vladimir.shur@urfu.ru (V.S.) \\ 3 CICECO-Aveiro Institute of Materials, Department of Physics, University of Aveiro, \\ 3810-193 Aveiro, Portugal \\ * Correspondence: kholkin@ua.pt (A.K.); igor.lubomirsky@weizmann.ac.il (I.L.)
}

Received: 27 September 2020; Accepted: 15 October 2020; Published: 19 October 2020

\begin{abstract}
Surface pyroelectricity and piezoelectricity induced by water incorporation during growth in $\alpha$-glycine were investigated. Using the periodic temperature change technique, we have determined the thickness $(\sim 280 \mu \mathrm{m})$ of the near surface layer (NSL) and its pyroelectric coefficient $\left(160 \mathrm{pC} /\left({\left.\mathrm{K} \times \mathrm{cm}^{2}\right)}^{2}\right.\right.$ at $\left.23^{\circ} \mathrm{C}\right)$ independently. The thickness of NSL remains nearly constant till $60^{\circ} \mathrm{C}$ and the pyroelectric effect vanishes abruptly by $70{ }^{\circ} \mathrm{C}$. The piezoelectric effect, $0.1 \mathrm{pm} / \mathrm{V}$ at $23^{\circ} \mathrm{C}$ measured with an interferometer, followed the same temperature dependence as the pyroelectric effect. Abrupt disappearance of both effects at $70^{\circ} \mathrm{C}$ is irreversible and suggests that water incorporation to $\alpha$-glycine forms a well defined near surface phase, which is different form $\alpha$-glycine because it is polar but it too close to $\alpha$-glycine to be distinguished by $X$-ray diffraction (XRD). The secondary pyroelectric effect was found to be $<14 \%$ of the total, which is unexpectedly small for a material with a large thermal expansion coefficient. This implies that water incorporation infers minimal distortions in the host lattice. This finding suggests a path for the control of the piezoelectric and pyroelectric effects of the crystals using stereospecific incorporation of the guest molecules.
\end{abstract}

Keywords: surface pyroelectricity; surface piezoelectricity; $\alpha$-glycine

\section{Introduction}

The crystal surface symmetry and physical properties are very important in a wide variety of applications, for example: using a suitable substrate can control symmetry and epitaxial growth of thin films for various applications in microelectronics, microelectromechanical systems (MEMS), catalysis and more. It has been reported that some centrosymmetric crystals contain a near surface layer (NSL) with polar symmetry. These layers were detected by pyroelectric measurements [1-3] or suggested by theoretical calculations [4-6]. Piezoelectric properties of such layers are frequently detected by Piezoresponse Force Microscopy (PFM) that is, in particular, sensitive to surface piezoelectricity [7-9]. The most popular interpretation of the surface piezoelectricity was a polarization arising due to strain gradient at the surface, i.e., flexoelectric effect. It can be caused, for example, by the PFM tip, that may cause highly inhomogeneous stress/strain just below the contact [10]. However, it is very hard to find any reliable experimental report on surface piezoelectricity in dielectric centrosymmetric crystals, although every non-centrosymmetric layer should give a piezoelectric response. The main reason for the lack of surface piezoelectric measurements is that even an NSL with a large piezoelectric coefficient, 
$d_{i i}$, will give a small displacement, $u$, even at high applied voltage as can be explained as follows. If the electric capacity and conductivity of the NSL are similar to those of the bulk crystal, the electric field inside the sample is constant and equal to $U / T h_{s}$, where $U$ is the external applied voltage and $T h_{s}$ is the sample thickness including the thickness of the NSL. In this case, the piezoelectric displacement will be described by [11]:

$$
u=d_{22} \times U \times \frac{\delta_{s}}{T h_{s}}
$$

where $\delta_{s}$ is the NSL thickness and $d_{22}$ is the piezoelectric coefficient in the polar direction in polar monoclinic crystals (subscript " 2 " indicates that it is in $b$-direction of the crystal (010)). Since for most single crystals $\frac{\delta_{s}}{T h_{s}}$ is in the order of $10^{-5}$, one should apply tens of thousands of volts to get a measurable displacement. Since, $\delta_{s}$ is usually unknown, the value that can be deduced from the experiment is:

$$
d_{22} \times \delta_{s}=\frac{u T h_{s}}{U}
$$

One exception to the above claim is $\alpha$-glycine ( $\alpha$-gly), which has a monoclinic centrosymmetic space group $P 2_{1 / n}$ with four molecules per unit cell $\left(a=5.105 \AA, b=11.97 \AA, c=5.465 A, \alpha=\gamma=90^{\circ}\right.$, $\beta=111.7^{\circ}$ ). In contrast to inorganic crystals like $\mathrm{SrTiO}_{3}[12]$, where the expected NSL is $<2 \mathrm{~nm}$ thick, the thickness of the surface polar layer in $\alpha$-gly, like in other amino acid crystals, may exceed 100 microns $[1-3,13]$. The NSL is so thick in $\alpha$-gly because a reduction in symmetry arises due to a partial water incorporation during the crystal growth [2]. This makes $\alpha$-gly a very suitable candidate for the experimental verification of the existence of the surface piezoelectricity in crystals. Measuring the surface piezoelectricity in $\alpha$-gly may answer two important questions:

(a) What is the magnitude of the surface piezoelectric effect, which will allow discrimination between the primary and secondary contributions to pyroelectricity.

(b) Does the surface piezoelectric effect vanishes concurrently with the pyroelectric effect or persists for some time after the latter disappears with temperature? If yes, would it imply that the structure first loses polarity but remains for some time non-polar but non-centrosymmetric.

Here, we report the results of the direct measurements of the temperature dependence of the piezoelectric effect in single crystals of $\alpha$-gly using a Michelson-Morley laser interferometer.

\section{Materials and Methods}

The single crystals of $\alpha$-gly were grown by slow evaporation technique from aqueous solution using a $100 \mathrm{~mL}$ of deionized water (Merck Millipore, Burlington, MA, USA, $18.2 \mathrm{M} \Omega \mathrm{cm}$ at $23^{\circ} \mathrm{C}$ Type 1 water) and $30 \mathrm{gr}$ of the glycine powder (Sigma-Aldrich, St. Louis, MO, USA, $\geq 99 \%$ ) as described previously [1,2]. In order to achieve complete dissolution of glycine powder, the solution was heated up to $75-80{ }^{\circ} \mathrm{C}$ and stirred for $3 \mathrm{~h}$. After the solution became completely transparent, it was filtered through cotton wool to the crystallization dishes (about 30-35 mL per one dish) and left for 12-17 h. The as-grown crystals were transparent and had a typical for $\alpha$-gly natural habitus with a clearly expressed $\{010\}$ planes, which was earlier reported to exhibit pyroelectricity $[1,2]$. The typical thickness of the crystal was 3-10 mm.

The as-grown crystals were cut parallel to the top exposed $\{010\}$ face into plates. Then, silver paste electrodes (VS Electronic Vertriebs, Aschaffenburg, Germany) were applied on both, top (as-grown) and bottom (sliced), \{010\}-faces. Four batches of crystals were grown and examined. For the as-grown surfaces, less than 30 min elapsed between the removal of crystals from the growth solution and commencing the measurements. As a control, some of the crystals were cleaved parallel to $\{010\}$ faces at least $0.3 \mathrm{~mm}$ below the naturally grown surface.

The displacement (in response to the applied voltage) was measured with a single-beam Michelson-Morley interferometer [14-16] equipped with a SR830 lock-in amplifier (Stanford Research Systems Inc., Sunnyvale, CA, USA). A proportional-integral-derivative (PID) feedback system consisting 
of P-841.01 piezo actuator and E-709.SRG piezo controller (Physik Instrumente, Karlsruhe, Germany) was used for the working point stabilization against the slow changes of the optical path length. A heating stage made of titanium film as a heating element clamped between two copper plates of $30 \times 30 \times 1.5 \mathrm{~mm}^{3}$ size was utilized for the temperature-dependent measurements. The temperature stabilization system was based on Pt100 class B resistance temperature sensor (Heraues Nexensos, Kleinostheim, Germany), MB110 input-output programming module (Owen, Moscow, Russia), and ZUP60-3.5 programmable power supply (TDK-Lambda Corporation, Tokyo, Japan). The heating stage was mounted on a polytetrafluoroethylene base, thermally isolated from the rest of the system with polyurethane foam. The measurements were carried out under excitation signal with amplitude of 50-150 $\mathrm{V}$ and frequency of $6-8 \mathrm{kHz}$, which is high enough to eliminate interference from mechanical resonances and electromagnetic noise. Every data point was obtained by averaging at least three measurements of the displacement amplitude.

The surface pyroelectric coefficient and the thickness of the NSL were measured using the periodic temperature change (PTC) as described in refs. [2,11].

\section{Results and Discussions}

\subsection{Pyroelectric Measurements}

All as-grown $\{010\}$ faces of $\alpha$-gly displayed a transient, $<100 \mathrm{msec}$, pyroelectric current in response to switching $\mathrm{ON}$ the laser illumination of the surface (Figure 1a), while the cleaved $\{010\}$ faces did not generate a pyroelectric response. According the derivations given in ref. [11], the current $(j)$ decays with time as:

$$
j=j_{0} \times \operatorname{erf}\left(\sqrt{\frac{\delta_{s}^{2}}{4 \cdot D \cdot t}}\right)
$$

where $\mathrm{D}=0.05 \mathrm{~cm}^{2} / \mathrm{s}$ [17] is the thermal diffusion coefficient of $\alpha$-gly and $j_{0}$ is the current at $t=0$. Fitting the $j$ vs. $t$ dependence to $e r f$-function leads to the value of $\delta_{s}=288 \pm 25 \mu \mathrm{m}$ (Figure 1b), which matches the previous estimates [17]. The fact that the current-time response can be fitted with high degree of confidence to Equaion (3) at all temperatures implies (Figure 1a) that the polarization is constant within the surface layer. $\delta_{s}$ does not change noticeably with temperature upon heating to $60^{\circ} \mathrm{C}$, above which the pyroelectric response rapidly drops and at $70{ }^{\circ} \mathrm{C}$ vanishes completely, which implies that the surface layer is a well-defined structure. The temperature at which the pyroelectric response vanishes is highly reproducible with about $\pm 4{ }^{\circ} \mathrm{C}$ variations between the samples. One has to point out that according to these measurements, the NSL has well defined thickness, constant polarization throughout and well-defined decomposition temperature, which strongly suggests that NSL is, in fact, a well-defined phase. $j_{0}$ can be expressed as:

$$
j_{0}=\frac{F_{d} \times p}{C_{V} \times T h_{s}}
$$

where $F_{d}$ is the heating power at the surface of the sample (in $W$ ) and $C_{V}=1.545 \mathrm{~J} /\left(\mathrm{cm}^{3} \times \mathrm{K}\right.$ ) is the thermal capacitance per unit volume. From the value of $j_{0}$ we have deduced the total pyroelectric coefficient, $p$, (Figure 2b). In contrast to the thickness of the NSL, the pyroelectric coefficient decays gradually from $40{ }^{\circ} \mathrm{C}$ to zero at $70{ }^{\circ} \mathrm{C}$. One has to point out that the pyroelectric coefficient of the NSL is fairly large for a non-ferroelectric material, $\approx 1.6 \times 10^{-10} \mathrm{C} /\left(\mathrm{m}^{2} \times \mathrm{K}\right)$ at $35^{\circ} \mathrm{C}$. The direction of the pyroelectric effect is always negative, which, assuming polarization decays with temperature, suggests that the polarization is directed outward of the crystal. 
a)

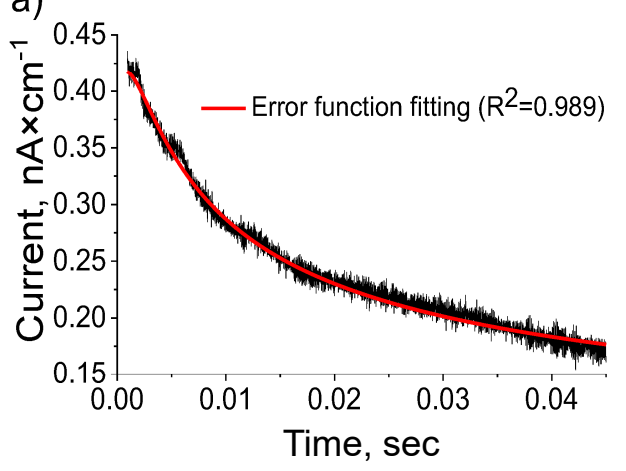

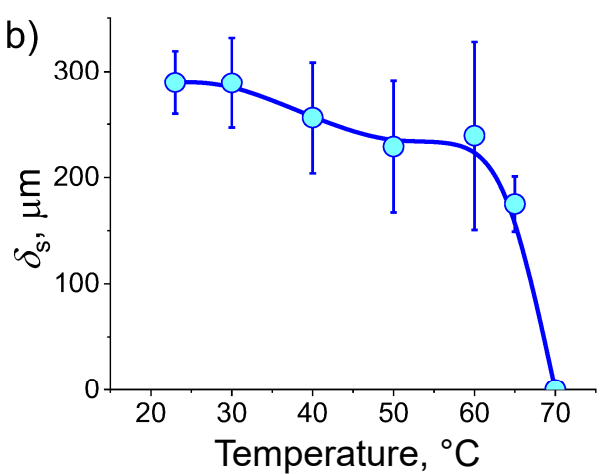

Figure 1. (a) Decay of the pyroelectric current with time $j(t)$, at $35^{\circ} \mathrm{C} ;(\mathbf{b})$ Temperature dependence of the NSL thickness $\delta_{s}$.
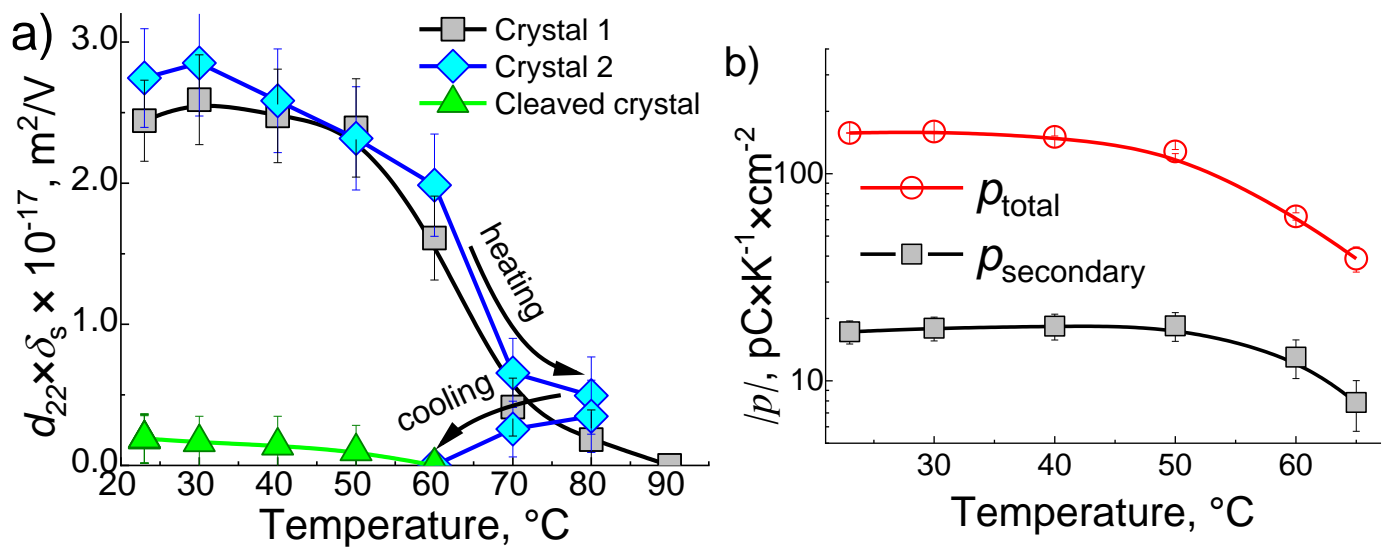

Figure 2. (a) The temperature dependence of product $d_{22} \times \delta_{s}$ for two as-grown $\{010\}$ surfaces of $\alpha$-gly. The graph for the cleaved crystal is shown for comparison; (b) The total and the secondary surface pyroelectric coefficients vs. temperature.

\subsection{Piezoelectric Measurements}

The as-grown $\{010\}$ faces of $\alpha$-glycine exhibit piezoelectric response, i.e., detectable surface displacement in response to applied voltage (Figure 2a). However, the cleaved $\{010\}$ faces do not show any displacement, which agrees with the absence of the pyroelectric response from them. The displacement of the as-grown $\{010\}$ faces in response to the applied voltage of 50-150 V was within a few pm range. The product $d_{22} \times \delta_{s}$ deduced from the displacement-temperature dependence was between $2 \times 10^{-17}$ and $3 \times 10^{-17} \mathrm{~m}^{2} / \mathrm{V}$. The temperature dependence of the $d_{22} \times \delta_{s}$ closely mirrored the temperature dependence of the pyroelectric coefficient $p$ : gradual decay starting from $40{ }^{\circ} \mathrm{C}$ and almost complete disappearance between 70 and $90^{\circ} \mathrm{C}$, indicating that there is a very narrow temperature range within which the NSL is still piezoelectric but already not pyroelectric. However, once the crystal reaches $70^{\circ} \mathrm{C}$, the decay in the piezoelectric effect becomes irreversible, even if the direction of the temperature change is reversed (Figure 2a, crystal 2). Using the value of $\delta_{s}$ deduced from the pyroelectric measurements, we have calculated the effective piezoelectric coefficient of NSL at each temperature. It is surprisingly small but still measurable, $d_{22}$, of $\sim 0.1 \mathrm{pm} / \mathrm{V}$. The reduction in the piezoelectric coefficient can be due to clamping of the surface layer by the rest of the crystal [18]. This effect is also responsible for the significant reduction in the piezoelectric coefficient of ferroelectric thin films as compared to their bulk counterparts [19].

It is of a particular interest to compare the total, $p$, and the secondary pyroelectric coefficient $p_{\text {sec }}=d_{22} \times \gamma_{2} \times Y_{2}$, where $\gamma_{2}=7 \times 10^{-5} \mathrm{~K}^{-1}$ is the thermal expansion coefficient of $\alpha$-gly in the [010] direction [17] and $Y_{2}=26 \mathrm{GPa}$ is the Young's modulus in the [010] direction [20]. While the total pyroelectric coefficient (measured at constant pressure) characterizes all contributions to the change 
in polarization, the secondary pyroelectric coefficient characterizes the contribution arising from the change in crystal dimensions due to thermal expansion. The difference between $p_{\text {primary }}=p-p_{\text {sec }}$ describes the changes to the overall polarization due to internal degrees of freedom, for the case of $\alpha$-gly, these are the changes in state of the incorporated water molecules. The results of the calculations show that the secondary pyroelectric coefficient is only $(12 \pm 2) \%$ of the total one, which is a particularly small, comparable only with that of $\mathrm{LiTaO}_{3}$, for which the thermal expansion coefficient in the polar direction is exceptionally small $\left(2 \times 10^{-6} \mathrm{~K}^{-1}\right)$ [21]. The relatively small secondary pyroelectric effect indicates that the polarization in the NSL is induced predominantly by the guest molecule, i.e., water, with minimum polar distortions introduced to the host lattice. As suggested earlier [1,2], the incorporation of water between the layer of $\alpha$-gly may be a satisfactory explanation of the weak piezoelectricity and small secondary pyroelectric effect. This also excludes a possibility that the polarity of the NSL comes from small inclusion of $\beta$-glycine, which is, indeed, polar [22].

In addition to providing a convincing evidence on the existence of surface piezoelectric effect in the NSL of $\alpha$-glycine and its nature, the data presented above suggest that stereospecific incorporation of small guest molecules (e.g., water) may take place without inducing distortions to the host lattice. Thereby, one can design a crystal with a large primary pyroelectric effect, induced by the guest, but with very low piezoelectric effect, because it originates from the host. This is very promising for the development of pyroelectric sensors in which the piezoelectric effect should be suppressed.

\section{Conclusions}

To conclude, we present a clear experimental evidence of the existence of surface piezoelectric effect in $\alpha$-glycine induced by the incorporation of water molecules during the crystal growth. These data complement earlier observations of the surface pyroelectricity in glycine and provide a way for the control of piezoelectric and pyroelectric effects of crystals using stereospecific incorporation of the guest molecules.

Author Contributions: I.L., D.E., M.L., S.D., A.K., V.S. conceived and designed the experiment; S.D., A.N. prepared the samples; S.D., A.U. performed the experiments; I.L., D.E., M.L., S.D. analyzed the data and prepared the original draft; A.K., V.S., A.N., A.U. reviewed and edited the draft. All authors have read and agreed to the published version of the manuscript.

Funding: This work was supported by the collaborative program of the Israeli Ministry of Science with the Russian Foundation for Basic Research, grant \#3-16492. This research was made possible in part by RFBR (Grant No. 19-52-06004 MNTI_a), and the Government of the Russian Federation (Act 211, Agreement 02.A03.21.0006). The work has been supported in part by the Ministry of Science and Higher Education of the Russian Federation under Project \#3.9534.2017/8.9. This work was developed within the scope of the project CICECO-Aveiro Institute of Materials, refs. UIDB/50011/2020 and UIDP/50011/2020, financed by national funds through the Portuguese Foundation for Science and Technology/MCTES.

Acknowledgments: The equipment of the Ural Center for Shared Use “Modern Nanotechnology" UrFU was used. I.L. expresses his gratitude to Estate of Olga Klein-Astrachan fund, grant \#721977.

Conflicts of Interest: The authors declare no conflict of interest.

\section{References}

1. Piperno, S.; Mirzadeh, E.; Mishuk, E.; Ehre, D.; Cohen, S.; Eisenstein, M.; Lahav, M.; Lubomirsky, I. Water Induced Pyroelectricity from Non-Polar Crystals of Amino Acids. Angew. Chem. Inter. Ed. 2013, 52, 6513-6516. [CrossRef]

2. Meirzadeh, E.; Sapir, L.; Cohen, H.; Cohen, S.R.; Ehre, D.; Harries, D.; Lahav, M.; Lubomirsky, I. Nonclassical Crystal Growth as Explanation for the Riddle of Polarity in Centrosymmetric Glycine Crystals. JACS 2016, 138, 14756-14763. [CrossRef]

3. Mishuk, E.; Weissbuch, I.; Lahav, M.; Lubomirsky, I. Pyroelectricity in Nonpolar Directions in Crystals: Enantiomeric Disorder and Surface Wetting in Racemic alpha-Amino-Acids. Cryst. Growth Des. 2014, 14, 3839-3848. [CrossRef] 
4. Dai, S.X.; Gharbi, M.; Sharma, P.; Park, H.S. Surface piezoelectricity: Size effects in nanostructures and the emergence of piezoelectricity in non-piezoelectric materials. J. Appl. Phys. 2011, 110. [CrossRef]

5. Georgescu, A.B.; Ismail-Beigi, S. Surface Piezoelectricity of (0001) Sapphire. Phys. Rev. Appl. $2019,11$. [CrossRef]

6. Munn, R.W. Microscopic Theory of Molecular-Crystal Surface Polarization, Piezoelectricity, and Pyroelectricity. J. Chem. Phys. 1994, 101, 5262-5266. [CrossRef]

7. Kholkin, A.; Bdikin, I.; Ostapchuk, T.; Petzelt, J. Room temperature surface piezoelectricity in $\mathrm{SrTiO}_{3}$ ceramics via piezoresponse force microscopy. Appl. Phys. Lett. 2008, 93. [CrossRef]

8. Tararam, R.; Bdikin, I.K.; Panwar, N.; Varela, J.A.; Bueno, P.R.; Kholkin, A.L. Nanoscale electromechanical properties of $\mathrm{CaCu}_{3} \mathrm{Ti}_{4} \mathrm{O}_{12}$ ceramics. J. Appl. Phys. 2011, 110. [CrossRef]

9. Andreeva, N.; Alikin, D.; Turygin, A.; Kholkin, A.L.; Shur, V.Y.; Filimonov, A.; Lessovaia, S. Temperature Dependence of Surface Polar State of $\mathrm{SrTiO}_{3}$ Ceramics Obtained by Piezoresponse Force Microscopy. Ferroelectrics 2015, 477, 1-8. [CrossRef]

10. Liu, W.Y.; Deng, F.; Xie, S.X.; Shen, S.P.; Li, J.Y. Electromechanical analysis of direct and converse flexoelectric effects under a scanning probe tip. J. Mech. Phys. Solids 2020, 142. [CrossRef]

11. Ehre, D.; Mirzadeh, E.; Stafsudd, O.; Lubomirsky, I. Pyroelectric Measurement of Surface Layer: The Case of Thin Film on Dielectric Substrate. Ferroelectrics 2014, 472, 41-49. [CrossRef]

12. Meirzadeh, E.; Christensen, D.V.; Makagon, E.; Cohen, H.; Rosenhek-Goldian, I.; Morales, E.H.; Bhowmik, A.; Lastra, J.M.G.; Rappe, A.M.; Ehre, D.; et al. Surface Pyroelectricity in Cubic SrTiO3. Adv. Mater. 2019, 31, e1904733. [CrossRef]

13. Meirzadeh, E.; Weissbuch, I.; Ehre, D.; Lahav, M.; Lubomirsky, I. Polar Imperfections in Amino Acid Crystals: Design, Structure, and Emerging Functionalities. Acc. Chem. Res. 2018, 51, 1238-1248. [CrossRef]

14. Mishuk, E.; Ushakov, A.; Makagon, E.; Cohen, S.R.; Wachtel, E.; Paul, T.; Tsur, Y.; Shur, V.Y.; Kholkin, A.; Lubomirsky, I. Electro-chemomechanical Contribution to Mechanical Actuation in Gd-Doped Ceria Membranes. Adv. Mater. Interfaces 2019, 6, 1801592. [CrossRef]

15. Mishuk, E.; Ushakov, A.D.; Cohen, S.R.; Shur, V.Y.; Kholkin, A.L.; Lubomirsky, I. Built-in bias in Gd-doped ceria films and its implication for electromechanical actuation devices. Sol. State Ion. 2018, 327, 47-51. [CrossRef]

16. Ushakov, A.D.; Yavo, N.; Mishuk, E.; Lubomirsky, I.; Shur, V.Y.; Kholkin, A.L. Electromechanical Measurements of Gd-Doped Ceria Thin Films by Laser Interferometry. Sino-Russ. Asrtu Symp. Adv. Mater. Process. Technol. 2016, 2016, 177-182. [CrossRef]

17. Meirzadeh, E.; Azuri, I.; Qi, Y.; Ehre, D.; Rappe, A.M.; Lahav, M.; Kronik, L.; Lubomirsky, I. Origin and structure of polar domains in doped molecular crystals. Nat. Commun. 2016, 7, 13351. [CrossRef]

18. Lefki, K.; Dormans, G.J.M. Measurement of Piezoelectric Coefficients of Ferroelectric Thin-Films. J. Appl. Phys. 1994, 76, 1764-1767. [CrossRef]

19. Kholkin, A.L.; Wutchrich, C.; Taylor, D.V.; Setter, N. Interferometric measurements of electric field-induced displacements in piezoelectric thin films. Rev. Sci. Instr. 1996, 67, 1935-1941. [CrossRef]

20. Azuri, I.; Meirzadeh, E.; Ehre, D.; Cohen, S.R.; Rappe, A.M.; Lahav, M.; Lubomirsky, I.; Kronik, L. Unusually Large Young's Moduli of Amino Acid Molecular Crystals. Angew. Chem. Int. Ed. Engl. 2015, 54, 13566-13570. [CrossRef]

21. Lang, S.B. Pyroelectricity: From ancient curiosity to modern imaging tool. Phys. Today 2005, 58, 31-36. [CrossRef]

22. Guerin, S.; Stapleton, A.; Chovan, D.; Mouras, R.; Gleeson, M.; McKeown, C.; Noor, M.R.; Silien, C.; Rhen, F.M.F.; Kholkin, A.L.; et al. Control of piezoelectricity in amino acids by supramolecular packing. Nat. Mater. 2018, 17, 180-186. [CrossRef] [PubMed]

Publisher's Note: MDPI stays neutral with regard to jurisdictional claims in published maps and institutional affiliations. 